\section{Eco.Mat}

Cúcuta-Colombia

Vol. 6

No. 1

Enero-Diciembre 2015 ISSN 1794-8231

ISSN 2462 - $8794 \mathrm{Web}$

PP: $22-33$

\title{
Aprendizaje y formación por competencias del pensamiento numérico
}

\section{Learning and formation for competitions of the numerical thought}

\author{
Rosa Virginia Hernández ${ }^{1}$, Luis Fernando Mariño ${ }^{2}$, José Orlando Cañas ${ }^{3}$
}

Forma de citar: R. V. Hernández, L. F. Mariño, J. O. Cañas, Aprendizaje y formación por competencias del pensamiento numérico, Eco.Mat. 2015; 6(1): 22-33.

Recibido:

Octubre 22 de 2014

Aceptado:

Diciembre 06 de 2014

Universidad Francisco de Paula Santander, correo: rosavirginiah@gmail.com

Universidad Francisco de Paula Santander, correo: fermarin3@gmail.com

Universidad Francisco de Paula Santander, correo: joseorlandocanas@msn.com

\section{RESUMEN:}

Describir las prácticas pedagógicas desarrolladas por el profesor de matemáticas entorno al aprendizaje de la competencia numérica, son la base para un adecuado desempeño en el ejercicio de la docencia y la dirección educativa, de este modo los sistemas educativos de cada país deben centrar la enseñanza de las matemáticas hacia habilidades y procesos que les permita a los estudiantes adquirir la capacidad de conocimiento, entendimiento y crítica entorno a la competencia numérica de manera flexible, para formular proposiciones matemáticas, desarrollo de estrategias útiles hacia la manipulación de los números, realizar operaciones y resolver problemas. Para el desarrollo de la investigación, se diseñó un instrumento tipo escala Likert con 26 ítems aplicado a 80 estudiantes de grado séptimo en la Institución Educativa Julio Pérez Ferrero, sedes Cundinamarca y Nuevo Horizonte en la ciudad de Cúcuta. Los resultados del instrumento, permiten concluir que el profesor solo tiene en cuenta el contenido programático, su criterio y experiencia para desarrollar las clases. Las dimensiones de aprendizaje en torno a la planeación, desarrollo y mejora de las prácticas pedagógicas hacia el aprendizaje de las matemáticas, especialmente la competencia numérica, juega un papel importante el factor evaluativo centrándose en cómo los estudiantes pueden utilizar lo que han aprendido en situaciones de la vida cotidiana.

Palabras claves: Práctica Pedagógica, Pensamiento Numérico, Competencia numérica

\section{SUMMARY:}

Describing the pedagogic practices developed by the professor of mathematics across of the numerical competition, the base for an adequate performance in the exercise of teaching and the educational address are, the educational systems out of every country to the learning in this way the teaching of the mathematics toward abilities that you allow to the students and processes must center to acquire the capability of knowledge, understanding and criticism surroundings to the numerical competition of flexible way, to formulate mathematical propositions, development of useful strategies toward the manipulation of numbers, to accomplish operations and to solve problems. For the development of investigation, type designed an instrument itself climb Likert with 26 applied items 80 students willingly seventh at the Institución Educativa Julio Pérez Ferrero, seats Cundinamarca and Nuevo Horizonte at the city of Cúcuta. The results of the instrument, they allow concluding that the very professor takes into account 
the programmatic contents, his opinion and experience to unroll classrooms. The learning dimensions about planning, development and improvement of the pedagogic practices toward the learning of mathematics, specially the numerical competition, play an important role the factor evaluativo becoming centered in how the students can utilize what they have learned in situations of the quotidian life.

Key words: Pedagogic practice, Numerical Pensamiento, numerical Competencia

\section{INTRODUCCIÓN}

Una de las grandes falencias hacia el aprendizaje de las matemáticas es la falta de una práctica docente coherente y actualizada que le permita al niño escolar despertar su interés de aprendizaje, junto a otros factores que inciden como grupos numerosos en las aulas, falta de recursos, problemas del entorno social donde se desenvuelve el niño y su familia, entre otros. Así lo afirman investigadores como Ruiz, L. L. W., y Pérez, M. I. R. (2014). Además, se debe tener en cuenta que debido a la gran crisis económica, los procesos de flexibilización y la precarización de las relaciones laborales muchos profesionales tanto jóvenes como adultos se están inclinando a ejercer la docencia, siendo una de las profesiones reconocidas con un muy bajo salario pero estable y con una serie de beneficios sociales todavía vigentes para los empleados estatales (Alliaud \& Davini, 1997; Birgin, 1999).

Sin embargo, aunque algunos profesores intentan adoptar y poner en práctica diversas teorías en su trabajo cotidiano, no es raro ver a muchos que se muestran escépticos o incluso no le ven la importancia o aplicabilidad fundamentada para que el estudiante adquiera su aprendizaje ya que los contenidos se encuentran en los libros considerando como irrelevantes teorías destacadas y comprobadas como significativas en el proceso de enseñanza aprendizaje. (Orton, 1990, p. 12). Por lo anterior, muchos de los docentes no tienen un sentido de pertenencia y responsabilidad para aplicar sus prácticas pedagógicas hacia un aprendizaje significativo y en mucho de los casos como lo afirma Ruiz, L. L. W., y Pérez, M. I. R. (2014, p. 2):

La realidad hacia el aprendizaje de las matemáticas que se ha venido presentado a través de los años por parte de los alumnos en todos los niveles educativos, es un sentimiento de rechazo, odio, miedo, ansiedad, frustración, bajo rendimiento, así como desmotivación por el hecho de que la asignatura se les haga demasiado compleja, aburrida o en el peor de los casos por el mal desempeño del docente dentro del aula.

Hacia la enseñanza de las matemáticas, se espera que el docente incorpore sus experiencias y saberes en estrategias didácticas bajo las condiciones pedagógicas (curriculares, didácticas $\mathrm{y}$ evaluativas) permitiendo a sus estudiantes propiciar escenarios dinámicos en torno a trabajos en equipo, espacios para lograr diálogos de intercambio conceptual entre estudiantes y el profesor con el objeto de lograr aprendizajes significativos, integrando el contexto escolar (institucional) y extraescolar (sociocultural) de los estudiantes. (MEN, 2006).

De este modo, los sistemas educativos de cada país deben concentrarse en las habilidades y en aquellos procesos que les den a los jóvenes el acceso al conocimiento, para entender, criticar y transformarlo. De ahí que la enseñanza de las matemáticas con la del español ocupen un lugar estratégico en la formación diseñada por 
Eco.Mat

Cúcuta-Colombia

Vol. 6

No. 1

Enero-Diciembre 2015 ISSN 1794-8231

ISSN 2462 - $8794 \mathrm{Web}$ PP: 22-33
Aprendizaje y formación por competencias del pensamiento numérico

los currículos de diversos países, incluyendo una participación sustancial en la carga horaria semanal (Terigi \& Wolman, 2007). Asimismo, la relevancia de la formación en la primera infancia ha crecido, relacionada con el deseo de preparar mejor a los niños para la escuela con la finalidad de asegurar su éxito escolar (Myers, 1999).

Bruno (1997), presenta las principales ideas teóricas, conclusiones e implicaciones didácticas de una investigación realizada con alumnos de 12-13 años de edad. Tal estudio concluye que si los docentes abandonan la dimensión contextual en la enseñanza de los números negativos, los estudiantes quedan sin los significados que realmente tienen en la vida real, y se produciría un cambio en el tipo de enseñanza numérica que hasta entonces han recibido los alumnos. Añade que algunas dificultades que surgen en la enseñanza de los números negativos son consecuencia del conocimiento previo sobre los positivos, y que un trabajo específico con los positivos las evitaría o disminuiría.

Teniendo en cuenta las situaciones problemas anteriormente mencionados y su respectiva justificación, a continuación se plantea la siguiente pregunta a nivel investigativo: ¿Cuáles son las prácticas pedagógicas que desarrolla el docente de matemáticas hacia el aprendizaje de la competencia numérica? El objetivo que intenta dar respuesta a la pregunta planteada es el siguiente: Describir las prácticas pedagógicas que desarrolla el profesor de matemáticas hacia el aprendizaje del pensamiento numérico.

Para el desarrollo de la investigación, se realizó un instrumento tipo escala Likert con 26 ítems aplicado a 80 estudiantes de grado séptimo en la Institución Educativa Julio Pérez Ferrero, sedes Cundinamarca y Nuevo Horizonte, cuyo soporte teórico es la guía 31 actualizaciones 2012 "Evaluación de competencias para el ascenso o reubicación de nivel salarial en el escalafón docente y directivos docentes regidos por el decreto ley 1278 de 2002 "; se parte de la reflexión pedagógica (currículo, didáctica y evaluación), $\mathrm{y}$ los espacios en donde se reconocen las problemáticas generales de la enseñanza y el aprendizaje (diseño de situaciones educativas, articulación de contextos del estudiante a la práctica educativa e integración del desarrollo del estudiante a la práctica educativa. (MEN, 2012).

En este sentido, para que el aprendizaje del niño sea significativo, el docente debe emplear técnicas y métodos que le faciliten asimilar el contenido programático y que los resultados sean satisfactorios para el docente, educando y su entorno; sin embargo, no es $\tan$ corriente ofrecer alternativas concretas y aplicables en el aula, que puedan sustituir el viejo aprendizaje basado en la simple memorización y a la enseñanza reducida a la mera aplicación de fórmulas memorísticas y pasivas, que trae como consecuencia, una enseñanza descontextualizada que no favorece la producción de conocimiento. (Viloria, N. \& Godoy G. 2010. p. 99).

Por tal razón, el desarrollo de la práctica pedagógica le permite planificar actividades donde se promueva el aprendizaje significativo y se apliquen estrategias adecuadas para la enseñanza de la matemática, especialmente hacia los números enteros. Considerando esta actividad como el proceso mediante el cual se logra combinar actividades y recursos que le permitan al docente atraer la atención la resolución de problemas, entre otros aspectos en el desarrollo de un contenido programático. El docente debe poseer una clara visión de los conocimientos para que el uso de estrategias didácticas dentro del aula permita al estudiante abordar el aprendizaje; puesto que la responsabilidad fundamental corresponde al docente que tiene la misión de formarlo, es importante que éste oriente a sus educandos, los incentive, despertando su iniciativa, sus ideas y tiene el deber de capacitarse permanentemente. (Viloria, N. \& Godoy G. 2010. P. 99). del grupo, incentivar la participación en 


\section{Materiales y Métodos}

Para el desarrollo de la investigación, se aplicó el enfoque cuantitativo, pues este se basa en el uso de técnicas estadísticas para conocer ciertos aspectos de interés sobre la población que se estudia, Peñuelas (2010, p.32), señala que el método cuantitativo se centra en los hechos o causas del fenómeno social, con escaso interés por los estados subjetivos del individuo. Este método utiliza el cuestionario, inventarios y análisis demográficos que producen números, los cuales pueden ser analizados estadísticamente para verificar, aprobar o rechazar las relaciones entre las variables definidas operacionalmente, además regularmente la presentación de resultados de estudios cuantitativos viene sustentada con tablas estadísticas, gráficas y un análisis numérico.

Para el diseño de la investigación, se diseñó y aplicó la encuesta escala Likert a ochenta (80) estudiantes de los grados séptimo con la finalidad de conocer la aptitud de aprendizaje que perciben los estudiantes entorno a la práctica pedagógica aplicada por los docentes del área de matemáticas, y especialmente el conocimiento de la competencia numérica involucrada en el aprendizaje de los números enteros según los parámetros curriculares y evaluativos del MEN.

Antes de la ejecución del instrumento tipo escala Likert se llevó a cabo una prueba piloto en la que se sometió a prueba el instrumento mediante un estudio a pequeña escala con el fin de determinar la validez del método y el procedimiento respectivo con objeto de obtener información permitiendo mejorar cada uno de los ítems en cuanto a su redacción y comprensión acorde con el soporte teórico y el objetivo planteado para poder realizar el análisis respectivo en forma satisfactoria.

Para el análisis de la información, se utilizó el Software SPSS V21, ya que esta herramienta permite realizar todo un análisis descriptivo, extraer y analizar las variables subyacentes o factores que explican la configuración de las correlaciones dentro de un conjunto de variables observadas. El análisis factorial es una técnica utilizada para la reducción de información, identificando un pequeño número de factores. El registro se hizo respetando la naturaleza de la medición de cada una de las variables, así como también se seleccionaron las técnicas de análisis descriptivos adecuadas para cada una de ellas. En primer lugar se procedió a realizar el respectivo análisis estadístico descriptivo utilizando principalmente como técnicas de análisis las tablas de distribución de frecuencias simples y conjuntas (Pardo \& Ruiz, 2002).

\section{Población y Muestra}

La población objeto de estudio, corresponde a estudiantes de grado séptimo en la Institución Educativa Julio Pérez Ferrero, sedes Cundinamarca y Nuevo Horizonte. La selección de los participantes fue realizada mediante muestreo intencional y durante el desarrollo de este proceso se eligieron ochenta (80) estudiantes. Es importante manifestar que el muestreo intencional fue generando de acuerdo al requerimiento para el trabajo de la encuesta escala Likert. Este tipo de muestreo se caracteriza por un esfuerzo deliberado de obtener muestras "representativas" mediante la inclusión en la muestra de grupos supuestamente típicos.

El investigador selecciona directa e intencionadamente los individuos de la población. El caso más frecuente de este procedimiento el utilizar como muestra los individuos a los que se tiene fácil acceso (los profesores de las instituciones emplean con mucha frecuencia a sus propios alumnos). (Hernández S. R., Collado F. \& Baptista L. 2010)

\section{Resultados y Discusión}

A continuación se presentan los resultados desde el punto de vista analítico que se
Enero-Diciembre 2015 ISSN 1794-8231 ISSN 2462 - $8794 \mathrm{Web}$ PP: $22-33$ 
Eco.Mat

Cúcuta-Colombia

Vol. 6

No. 1

Enero-Diciembre 2015 ISSN 1794-8231 ISSN $2462-8794 \mathrm{We}$

PP: $22-33$
Aprendizaje y formación por competencias del pensamiento numérico

obtuvieron luego de aplicar las técnicas de análisis respectivas teniendo en cuenta el soporte teórico y la metodología planteada.

\section{Análisis Descriptivo}

La Tabla 1 muestra las distribuciones de frecuencia de los 26 ítems del cuestionario aplicado a los estudiantes, clasificado en tres dimensiones: Planeación, Desarrollo y Mejora. En ella se puede observar que la mayoría de los ítems se obtiene un puntaje alto en la categoría de la escala referida a "Siempre". Esto implica que los estudiantes perciben una marcada presencia de las características estudiadas en los docentes de matemáticas.

Tabla I. DIMENSIONES DEL APRENDIZAJE DE LA COMPETENCIA NUMÉRICA

\begin{tabular}{|c|c|c|c|c|c|c|c|}
\hline & & \multirow[t]{2}{*}{ Aspectos } & \multicolumn{5}{|c|}{ Frecuencia con que realiza la actividad } \\
\hline & & & Siempre & $\begin{array}{c}\text { Casi } \\
\text { siempre }\end{array}$ & $\begin{array}{l}\text { Algunas } \\
\text { Veces } \\
\end{array}$ & $\begin{array}{c}\text { Casi } \\
\text { nunca }\end{array}$ & Nunca \\
\hline \multirow{9}{*}{ 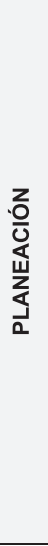 } & 1 & $\begin{array}{l}\text { El docente aplica prueba diagnóstica (repaso) al iniciar el } \\
\text { año escolar }\end{array}$ & $56,3 \%$ & $18,8 \%$ & $20,0 \%$ & $1,3 \%$ & $3,8 \%$ \\
\hline & 2 & $\begin{array}{l}\text { El docente utiliza diferentes tipos de materiales (textos o } \\
\text { guías) para el desarrollo de las clases }\end{array}$ & $56,3 \%$ & $27,5 \%$ & $12,5 \%$ & $3,8 \%$ & \\
\hline & 3 & $\begin{array}{l}\text { Los ejemplos usados en clase de matemáticas están } \\
\text { relacionados con aspectos del entorno donde vives }\end{array}$ & $11,3 \%$ & $37,5 \%$ & $26,3 \%$ & $15,0 \%$ & $10,0 \%$ \\
\hline & 4 & $\begin{array}{l}\text { El área de matemáticas desarrolla proyectos relacionado } \\
\text { con otras materias }\end{array}$ & $8,8 \%$ & $20,0 \%$ & $33,8 \%$ & $16,3 \%$ & $21,3 \%$ \\
\hline & 5 & $\begin{array}{l}\text { Para el desarrollo de la clase, el profesor se apoya en } \\
\text { recursos tecnológicos }\end{array}$ & $17,5 \%$ & $22,5 \%$ & $36,3 \%$ & $11,3 \%$ & $12,5 \%$ \\
\hline & 6 & $\begin{array}{l}\text { Para el desarrollo de la clase, el profesor se apoya en } \\
\text { investigaciones }\end{array}$ & $25,0 \%$ & $40,0 \%$ & $21,3 \%$ & $5,0 \%$ & $8,8 \%$ \\
\hline & 7 & $\begin{array}{l}\text { El profesor presenta actividades para trabajar en el aula en } \\
\text { forma individual }\end{array}$ & $38,8 \%$ & $40,0 \%$ & $17,5 \%$ & $1,3 \%$ & $2,5 \%$ \\
\hline & 8 & $\begin{array}{l}\text { El profesor señala cuales fueron las fallas en la evaluación } \\
\text { luego de calificarla }\end{array}$ & $56,3 \%$ & $21,3 \%$ & $12,5 \%$ & $5,0 \%$ & $5,0 \%$ \\
\hline & 9 & $\begin{array}{l}\text { El profesor se preocupa por aclarar las dudas surgidas } \\
\text { antes de las evaluaciones }\end{array}$ & $56,3 \%$ & $18,8 \%$ & $15,0 \%$ & $3,8 \%$ & $6,3 \%$ \\
\hline \multirow{12}{*}{ 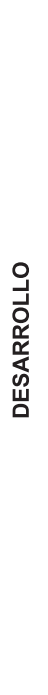 } & 10 & $\begin{array}{l}\text { El profesor presenta los temas de clase desvinculados de la } \\
\text { vida real }\end{array}$ & $10,0 \%$ & $8,8 \%$ & $35,0 \%$ & $36,3 \%$ & $10,0 \%$ \\
\hline & 11 & $\begin{array}{l}\text { El profesor relaciona los temas con los fenómenos sociales } \\
\text { y naturales que ocurren en el momento }\end{array}$ & $10,0 \%$ & $26,3 \%$ & $32,5 \%$ & $25,0 \%$ & $6,3 \%$ \\
\hline & 12 & El profesor hace la clase muy monótona y aburrida & $20,0 \%$ & $17,5 \%$ & $38,8 \%$ & $3,8 \%$ & $20,0 \%$ \\
\hline & 13 & $\begin{array}{l}\text { Las actividades realizadas en clase invitan a reflexionar } \\
\text { para hacer aportes sobre la temática desarrollada }\end{array}$ & $30,0 \%$ & $38,8 \%$ & $22,5 \%$ & $6,3 \%$ & $2,5 \%$ \\
\hline & 14 & $\begin{array}{l}\text { Durante el trabajo grupal el profesor acompaña aclarando } \\
\text { las dudas }\end{array}$ & $43,8 \%$ & $45,0 \%$ & $8,8 \%$ & $0 \%$ & $5,0 \%$ \\
\hline & 15 & $\begin{array}{l}\text { El profesor promueve la participación y la tiene en cuenta a } \\
\text { la hora de evaluar }\end{array}$ & $41,3 \%$ & $35,0 \%$ & $18,8 \%$ & $0 \%$ & $5,0 \%$ \\
\hline & 16 & $\begin{array}{l}\text { El profesor evalúa de manera continua y realiza } \\
\text { correcciones para ver donde fallaron }\end{array}$ & $32,5 \%$ & $43,8 \%$ & $17,5 \%$ & $3,8 \%$ & $2,5 \%$ \\
\hline & 17 & $\begin{array}{l}\text { El profesor invita al estudiante oportunamente a } \\
\text { autoevaluarse }\end{array}$ & $41,3 \%$ & $22,5 \%$ & $21,3 \%$ & $11,3 \%$ & $3,8 \%$ \\
\hline & 18 & $\begin{array}{l}\text { El profesor invita al grupo a practicar la co-evaluación en } \\
\text { los momentos indicados }\end{array}$ & $37,5 \%$ & $36,3 \%$ & $17,5 \%$ & $6,3 \%$ & $2,5 \%$ \\
\hline & 19 & $\begin{array}{l}\text { El profesor se retrasa en informar y soportar los resultados } \\
\text { de las evaluaciones }\end{array}$ & $21,3 \%$ & $15,0 \%$ & $33,8 \%$ & $22,5 \%$ & $7,5 \%$ \\
\hline & 23 & $\begin{array}{l}\text { El profesor considera los resultados de las evaluaciones } \\
\text { para implementar nuevas estrategias de recuperación }\end{array}$ & $36,3 \%$ & $41,3 \%$ & $18,8 \%$ & $0 \%$ & $3,8 \%$ \\
\hline & 24 & $\begin{array}{l}\text { Al profesor se le dificulta explicar con detalle el tema de } \\
\text { clase en un tiempo planificado }\end{array}$ & $46,3 \%$ & $31,3 \%$ & $15,0 \%$ & $5,0 \%$ & $2,5 \%$ \\
\hline \multirow{5}{*}{$\begin{array}{l}\overleftarrow{\alpha} \\
\text { o } \\
\text { 山ై }\end{array}$} & 20 & $\begin{array}{l}\text { Se desarrollan los contenidos programados para cada } \\
\text { periodo }\end{array}$ & $43,8 \%$ & $33,8 \%$ & $13,8 \%$ & $7,5 \%$ & $1,3 \%$ \\
\hline & 21 & $\begin{array}{l}\text { El profesor solo tiene en cuenta su criterio y experiencia } \\
\text { para desarrollar sus clases }\end{array}$ & $25,0 \%$ & $18,8 \%$ & $32,5 \%$ & $12,5 \%$ & $11,3 \%$ \\
\hline & 22 & $\begin{array}{l}\text { El profesor utiliza diferentes medios e instrumentos para } \\
\text { evaluar los contenidos }\end{array}$ & $22,5 \%$ & $25,0 \%$ & $35,0 \%$ & $8,8 \%$ & $8,8 \%$ \\
\hline & 25 & $\begin{array}{l}\text { Las actividades prácticas están relacionadas con los temas } \\
\text { vistos en clase }\end{array}$ & $53,8 \%$ & $30,0 \%$ & $13,8 \%$ & $1,3 \%$ & $1,3 \%$ \\
\hline & 26 & $\begin{array}{l}\text { El aprendizaje en Matemáticas le ha permitido resolver } \\
\text { situaciones de la vida diaria }\end{array}$ & $31,3 \%$ & $26,3 \%$ & $25,0 \%$ & $5,0 \%$ & $12,5 \%$ \\
\hline
\end{tabular}


La "planeación" que aplica el docente de matemáticas para el desarrollo del aprendizaje en el aula, aproximadamente un $56 \%$ de estudiantes evaluaron como "siempre" a las siguientes actividades: al inicio del año aplica una prueba diagnóstica, aplica diferentes materiales para el desarrollo de las clases, una vez presentada la evaluación realiza las correcciones y se preocupa por aclarar las dudas surgidas durante los temas orientados; sin embargo se presentó en promedio del $35 \%$ de estudiantes que evaluaron "algunas veces" las actividades como el apoyo del uso de recursos tecnológicos y el aprendizaje a nivel investigativo.

Hacia el "desarrollo" del aprendizaje de matemáticas se presentó un promedio del $40 \%$ evaluado como "siempre" por los estudiantes hacia los docentes en cuanto a la motivación hacia la participación, autoevaluación $y$ coevaluación como parte de su formación integral. Igualmente se refleja un $36,3 \%$ de los estudiantes que evaluaron como "casi nunca" el desarrollo del aprendizaje a través de temas vinculados con la vida real.

Para la "mejora" hacia el aprendizaje de las matemáticas se presentó un promedio del $43 \%$ evaluado como "siempre" en las actividades como el desarrollo de los contenidos programados en cada periodo, actividades prácticas relacionadas con los temas vistos y el aprendizaje de las matemáticas le ha permitido resolver situaciones de la vida real. Se evaluó como "algunas veces" en promedio por un $33 \%$ de estudiantes hacia las actividades en el cual el docente solo tiene en cuenta su criterio y experiencia para desarrollar sus clases y utiliza diferentes medios e instrumentos para evaluar los contenidos.

En la dimensión del Desarrollo, la categoría de "casi siempre", se destacan porcentajes alrededor del 45\% los ítems (14 y 16), donde los docentes se preocupan por aclarar dudas en clase, evaluar de manera continua los temas tratados en clase y haciendo un refuerzo conceptual cuando se presentan dificultades de aprendizaje. No obstante según los encuestados (46,3\%), se percibe que a los docentes se les dificulta explicar el tema en un tiempo determinado, postergando la clase a encuentros siguientes. Por último en la dimensión de "Mejora", los alumnos admiten en un $38,8 \%$ que los profesores a pesar de preocuparse por utilizar diferentes recursos tecnológicos y formas de orientar la clase, esta temática es monótona y aburrida. De igual forma, los estudiantes sugieren que los docentes deben tardar menos en entregar los resultados de las evaluaciones para trabajar y reforzar los puntos débiles detectados.

\section{Análisis Factorial}

El AF puede ejecutarse en cuatro etapas, (Pardo \& Ruiz, 2002): 1) El Cálculo de la matriz de correlaciones; 2) La extracción del número óptimo de factores; 3) La rotación de la solución para facilitar su interpretación y 4) la estimación de las puntuaciones de los sujetos en las nuevas dimensiones. A continuación se especifican y definen cada uno de los pasos siguiendo el procedimiento presentado por Hair y otros, (2002).

Los supuestos básicos subyacentes del AF son más de tipo conceptual que estadístico, la normalidad sólo es necesaria cuando se aplica una prueba estadística a la significación de los factores (Hair et al., 2001). Por otra parte es deseable, que exista correlación entre las variables, sin embargo, que esta no sea muy alta, pues se puede caer en la multicolinealidad que imposibilita la extracción de factores y en consecuencia la ejecución del AF.

Los resultados de la verificación de supuestos muestran un valor del determinante de 0,00002551, el KMO alcanza el valor de 0,604 y la prueba de esfericidad de Barlett arroja un chi cuadrado de $\chi^{2}=735,051$ y $\mathrm{p}=0,00$ (al ser el valor de $\mathrm{p}<0,05$ se rechaza la hipótesis de igualdad), estos niveles de las 
Eco.Mat

Cúcuta-Colombia

Vol. 6

No. 1

Enero-Diciembre 2015 ISSN 1794-8231

ISSN $2462-8794$ Web PP: 22-33

\section{Extracción de Factores}

Para la consideración del número óptimo de factores se toma como indicador importante la magnitud de los autovalores y el porcentaje de varianza explicada por cada factor. La Tabla 2 muestra la relación de autovalores y el porcentaje de varianza explicado por cada factor.

Como se muestra en la Tabla 2, se observan seis factores claramente identificados y que aportan en buena proporción a la explicación de la varianza del modelo. Los primeros tres factores explican aproximadamente el 37\% de la varianza. Los factores restantes explican aproximadamente el 19\% restante. La solución de seis factores indica la adecuación de la aplicación de esta técnica de reducción de la dimensionalidad. El total de varianza explicada es aproximadamente el 56\%, lo cual se considera muy óptimo.

La tabla 2 muestra el primer factor que explica un $21,5 \%$ de la varianza está compuesto por las variables $(16,8,23,17,15,9)$.El segundo factor, explica aproximadamente el 7,8\% de la varianza y está formado por las variables (1, 13, 3, 4, 26). El tercer factor explica el 7,5\% de la varianza está compuesto por las variable $(6,10,22)$. El cuarto factor que explica un $7,3 \%$ de la varianza total, lo componen las variables $(12,19,18,7,14,20)$. El quinto factor que explica un $6,2 \%$ de la varianza total, lo componen las variables $(24,21)$. El sexto y último factor que explica un $5,5 \%$ de la varianza total, lo componen las variables $(2,5,11,25)$. El peso de cada variable y su identificación en función del factor del cual forma parte se expresa en la Tabla 3.

Tabla II. VARIANZA TOTAL EXPLICADA

\begin{tabular}{|c|c|c|c|c|c|c|}
\hline \multirow[b]{2}{*}{ Componente } & \multicolumn{3}{|c|}{ Autovalores iniciales } & \multicolumn{3}{|c|}{$\begin{array}{c}\text { Sumas de las saturaciones al cuadrado de la } \\
\text { extracción }\end{array}$} \\
\hline & Total & $\begin{array}{l}\text { \%o de la } \\
\text { varianza }\end{array}$ & 96 acumulado & Total & $\begin{array}{l}96 \text { de la } \\
\text { varianza }\end{array}$ & 96 acumulado \\
\hline & 5,610 & 21,576 & 21.576 & 5,610 & 21,576 & 21,576 \\
\hline 2 & 2,050 & 7,885 & 29,461 & 2,050 & 7,885 & 29,461 \\
\hline 3 & 1,969 & 7.572 & 37,033 & 1,969 & 7,572 & 37,033 \\
\hline 4 & 1.898 & 7,301 & 44,334 & 1,898 & 7,301 & 44,334 \\
\hline 5 & 1,621 & 6,236 & 50,569 & 1,621 & 6,236 & 50,569 \\
\hline 6 & 1,443 & 5,551 & 56,121 & 1,443 & 5,551 & 56,121 \\
\hline 7 & 1,174 & 4,515 & 60,636 & & & \\
\hline 8 & 1.112 & 4,278 & 64,913 & & & \\
\hline 9 & 1,082 & 4,160 & 69,073 & & & \\
\hline 10 & .970 & 3,730 & 72,803 & & & \\
\hline 11 & .859 & 3,302 & 76,105 & & & \\
\hline 12 & .804 & 3,091 & 79,196 & & & \\
\hline 13 & .709 & 2,729 & 81,925 & & & \\
\hline 14 & .669 & 2,574 & 84,499 & & & \\
\hline 15 & .559 & 2,150 & 86,649 & & & \\
\hline 16 & .533 & 2,051 & 88,701 & & & \\
\hline 17 & 490 & 1,885 & 90,586 & & & \\
\hline 18 & .462 & 1.776 & 92,362 & & & \\
\hline 19 & .362 & 1,393 & 93,756 & & & \\
\hline 20 & .347 & 1,333 & 95,089 & & & \\
\hline 21 & .303 & 1,167 & 96,255 & & & \\
\hline 22 & .274 & 1.054 & 97.309 & & & \\
\hline 23 & .234 & .901 & 98,211 & & & \\
\hline 24 & .195 & .751 & 98,961 & & & \\
\hline 25 & 161 & .620 & 99,581 & & & \\
\hline 26 & 109 & .419 & 100,000 & & & \\
\hline
\end{tabular}

\section{Estructura Factorial}

Con la finalidad de encontrar una solución factorial, se revisa el peso de cada variable en cada uno de los factores (Tabla 2). 
Tabla III. MATRIZ DE ESTRUCTURA

\begin{tabular}{|c|c|c|c|c|c|c|}
\hline \multirow{2}{*}{\multicolumn{7}{|c|}{ Matriz de estructura }} \\
\hline & & & & & & \\
\hline & 1 & 2 & 3 & 4 & 5 & 6 \\
\hline 16. Evaluacion continua y corrige & 0,758 & 0,156 & 0,231 & 0,309 & $-0,004$ & 0,009 \\
\hline 8. Socializacion de fallas & 0,682 & 0,260 & 0,259 & 0,118 & 0,003 & 0,039 \\
\hline 23. Resultados/Estrategias & 0,672 & 0,551 & 0,472 & 0,374 & $-0,132$ & $-0,023$ \\
\hline 17. Invita a la auto-evaluacion & 0,667 & $-0,047$ & 0,421 & 0,132 & $-0,089$ & 0,240 \\
\hline 15. Promueve participacion & 0,649 & 0,535 & 0,340 & 0,301 & $-0,216$ & $-0,226$ \\
\hline 9. Aclarar dudas & 0,640 & 0,151 & 0,194 & 0,461 & 0,237 & $-0,036$ \\
\hline 1. Prueba diag & 0,140 & 0,686 & $-0,108$ & 0,100 & $-0,059$ & 0,118 \\
\hline 13. Invita a la reflexio & 0,435 & 0,645 & 0,205 & 0,481 & $-0,178$ & 0,032 \\
\hline 3. Relacion con el entorno & 0,050 & 0,615 & 0,441 & 0,278 & 0,073 & $-0,044$ \\
\hline 4. Proyectos con otras & 0,117 & 0,584 & 0,400 & $-0,035$ & 0,410 & $-0,076$ \\
\hline 26. Resolucion de problema & 0,456 & 0.563 & 0.119 & 0,158 & $-0,035$ & $-0,194$ \\
\hline 6. Investigaciones & 0,433 & 0,213 & 0,739 & 0,214 & 0,110 & 0,137 \\
\hline 10. Temas desvinculac & $-0,179$ & $-0,033$ & $-0,679$ & $-0,011$ & 0,070 & $-0,012$ \\
\hline 22. Diferen & 0,432 & 0,221 & 0,498 & 0,372 & $-0,464$ & $-0,211$ \\
\hline 12. Clase monotona y aburrida & 0,297 & 0,413 & 0,008 & 0,725 & $-0,117$ & $-0,121$ \\
\hline 19. Retraso en la entrega de notas & 0,056 & 0,167 & $-0,161$ & 0,635 & 0,333 & 0,123 \\
\hline 18. Invita a la co-evaluacion & 0.158 & -0.049 & 0.320 & 0.559 & -0.249 & -0.074 \\
\hline 7. Actividad it & 0,333 & $-0,106$ & 0,399 & 0,557 & 0,184 & 0,210 \\
\hline 14. Acompaña a los trabajos en grupo & 0,476 & 0,327 & 0,094 & 0,507 & $-0,001$ & $-0,043$ \\
\hline 20. Desarr & 0,185 & 0,162 & 0,188 & 0,436 & $-0,039$ & 0,323 \\
\hline 24. Dificuli & 0,032 & 0,067 & 0,095 & 0,142 & 0,752 & 0,093 \\
\hline 21. Solar & $-0,026$ & 0,073 & 0,078 & 0,079 & $-0,700$ & 0,110 \\
\hline 2. Tipos de materiales & 0,183 & 0,122 & 0,315 & 0,048 & $-0,028$ & 0,727 \\
\hline 5. Recursos tecnologicos & 0,139 & 0,080 & 0,329 & $-0,071$ & 0,099 & $-0,610$ \\
\hline & 0,182 & 0,431 & 0,489 & 0.240 & $-0,153$ & $-0,498$ \\
\hline 5. Relac & $462+2,+2$ & 0.146 & $0.319+2,+3>0$ & 0.240 & 0.284 & 0.481 \\
\hline
\end{tabular}

Las cargas factoriales representan el peso de cada una de las variables en los factores obtenidos en la solución factorial. Se considera que la variable observada satura mejor en el factor cuya carga factorial es más alta. La Tabla 2 muestra, las cargas factoriales de cada variable observada en que cada uno de los factores obtenidos, se resalta el mayor peso factorial en el factor donde satura la variable. Está tabla nos facilita la obtención de la estructura factorial en función de las variables observadas. Se obtiene así una configuración hexafactorial, organizada según la Tabla 2, en ella se observa que los pesos factoriales oscilan entre 0,436 y 0,758 .

\section{Denominación de los Factores}

Una vez obtenida la solución factorial que satisface los supuestos y exigencias de la técnica, el investigador debe atribuir el significado a los hallazgos encontrados. "El proceso implica la interpretación sustantiva del patrón de cargas factoriales para las variables, incluidos sus signos, en un esfuerzo por denominar cada uno de los factores", (Hair y otros, 2002, p113). Para la concepción de la etiqueta del factor se consideran todas las variables, especialmente las que tienen un mayor peso o ponderación en la estructura del factor.

El primer factor denominado Evaluación, incluye la actuación del docente frente a los hechos cotidianos en el aula de clase, frente a este evento se debe evaluar de forma continua parapoderaclarardudasluegodelas respectivas correcciones. Con base en la auto-evaluación, se pueden establecer estrategias para mejorar $\mathrm{y}$ crear un ambiente participativo con los estudiantes. Sin embargo, si nos ponemos a hacer una comparación de este proceso con las pruebas evaluativas de los grupos de expertos en matemáticas (Mathematic Expert Group, MEG) en el Proyecto PISA 2003, que son los responsables de estudiar las muestras de ítems, revisar sus enunciados, analizar los resultados de estudios piloto y proponer los ítems finales, en coordinación con el Australian Council of Educational Research (HACER) y el instituto Freudenthal; podemos afirmar que los docentes están desfasados a nivel evaluativo. (Rico, 2004).

Los docentes de las escuelas y colegios aún no establecen en forma clara y definida a nivel evaluativo que las competencias en matemáticas se consideran la parte principal 


\begin{tabular}{|c|c|c|c|}
\hline Eco.Mat & & & \\
\hline Cúcuta-Colombia & \multirow{2}{*}{\multicolumn{3}{|c|}{ Aprendizaje y formación por competencias del pensamie }} \\
\hline \multicolumn{3}{|l|}{ Vol. 6} & \\
\hline \multicolumn{4}{|l|}{ No. 1} \\
\hline nero-Diciembre 2015 & \multicolumn{3}{|c|}{ Tabla IV. DENOMINACIÓN DE LOS FACTORES } \\
\hline ISSN 1794-8231 & FACTOR & Denominación & Variables \\
\hline \multirow{8}{*}{$\begin{array}{l}\text { SN } 2462-8794 \text { Web } \\
\text { PP: } 22-33\end{array}$} & & & \multirow{3}{*}{$\begin{array}{l}\text { 16. Evaluacion continua y corrige } \\
\text { 8. Socializacion de fallas } \\
\text { 23. Resultados/Estrategias } \\
\text { 17. Invita a la auto-evaluacion } \\
\text { 15. Promueve participacion } \\
\text { 9. Aclarar dudas }\end{array}$} \\
\hline & 1 & EVALUACION & \\
\hline & & & \\
\hline & 2 & ENTORNO & $\begin{array}{l}\text { 1. Prue ba diagnostica } \\
\text { 13. Invita a la reflexion y aportes } \\
\text { 3. Relacion con el entorno } \\
\text { 4. Proy ectos con otras materias } \\
\text { 26. Resolucion de problemas reales } \\
\end{array}$ \\
\hline & 3 & PROYECTOS & $\begin{array}{l}\text { 6. Irvestigaciones } \\
\text { 10. Temas desvinculados de la vida real } \\
\text { 22. Diferentes medios e instrumentos }\end{array}$ \\
\hline & 4 & PRACTICA PEDAGOGICA & $\begin{array}{l}\text { 12. Clase monotona y aburrida } \\
\text { 19. Retraso en la entrega de notas } \\
\text { 18. Invita a la co-evaluacion } \\
\text { 7. Actividad individual } \\
\text { 14. Acompaña a los trabajos en grupo } \\
\text { 20. Desarrollo del contenido }\end{array}$ \\
\hline & 5 & DEBIUDADES & $\begin{array}{l}\text { 24. Dificultad para explicar } \\
\text { 21. Solamente su criterio }\end{array}$ \\
\hline & 6 & RECURSOS & $\begin{array}{l}\text { 2. Tipos de materiales } \\
\text { 5. Recursos tecnologicos } \\
\text { 11. Relacion Tema-Actualidad } \\
\text { 25. Relacion Evaluacion/Clase }\end{array}$ \\
\hline
\end{tabular}

de la preparación educativa $\mathrm{y}$, por ello, la evaluación en matemáticas es un componente esencial del programa PISA. El foco de evaluación en el programa se centra en cómo los estudiantes pueden utilizar lo que han aprendido en situaciones usuales de la vida cotidiana y no sólo, ni principalmente, en conocer cuáles contenidos del currículo han aprendido. (Romero, 2004).

El segundo factor denominado Entorno, está conformado por acciones que el docente implementa para relacionar lo aprendido en clase con el contexto. Concretamente hace un diagnóstico de conocimientos previos e invita a la reflexión para así, aportar los nuevos conocimientos. Con esta información nueva, se procede a identificar situaciones problémicas reales, concernientes al tema y poderlas solucionar. Se busca en el estudiante un desarrollo óptimo de la competencia numérica para hacerla útil, incluso en otras materias como parte de un proyecto.

Los porcentajes que se obtuvieron en la respectiva prueba en relación a la vinculación de los temas con la vida real, los ejemplos que el docente aplica relacionados con el entorno fueron evaluados en el nivel de "casi siempre" con porcentajes inferiores al 50\%; esto es preocupante; según el MEN (2006), formular, plantear, transformar y resolver problemas a partir de situaciones de la vida cotidiana, de las otras ciencias y de las matemáticas mismas, requiere que el docente analice sus prácticas pedagógicas y establezca un vínculo permanente entre el contenido curricular, los estándares de las competencias y el método de evaluación como mínimo asociado a las pruebas SABER en Colombia. Este proceso general requiere del uso flexible de conceptos, procedimientos y diversos lenguajes para expresar las ideas matemáticas pertinentes y para formular, reformular, tratar y resolver los problemas asociados a dicha situación. Estas actividades también integran el razonamiento, en tanto exigen formular argumentos que justifiquen los análisis y procedimientos realizados y la validez de las soluciones propuestas.

El tercer factor denominado Proyectos, hace referencia a los objetivos que traza el docente para que sus estudiantes los cumplan a través de evidencias como proyectos, investigaciones y trabajos de campo. Estos compromisos no deben estar desvinculados de la vida cotidiana para que los estudiantes los ejecuten con motivación, convencidos de que están adquiriendo un conocimiento útil de cara al futuro, en su vida personal y profesional. Si estos compromisos no se cumplen los estudiantes seguirán teniendo un nivel de rechazo, odio, miedo, ansiedad, frustración, bajo rendimiento, así como la desmotivación hacia el aprendizaje de las 
matemáticas debido a la forma compleja, aburrida, tradicional y mecánica como el docente la está orientando; acorde con el pensamiento de Ruiz, L. L. W., y Pérez, M. I. R. (2014).

El cuarto factor, denominado Practica Pedagógica, está relacionado con las acciones del docente dentro del aula, percibidas por los alumnos. A favor de los profesores está el cumplimiento de los contenidos, el acompañamiento en los trabajos en grupo $\mathrm{y}$ que conceden espacios para el trabajo individual. En contra, los estudiantes recomiendan no hacer la clase tan monótona, entregar los resultados en menos tiempo y acudir a la co-evaluación como parte de la nota final, creando un ambiente reflexivo a través de los pares. Todas estas actividades están relacionadas con la enseñanza tradicional del cual hoy en día no es la más adecuada debido a que se requiere de la incorporación de las herramientas tecnológicas (Tic) para que se pueda motivar el aprendizaje y especialmente se le enfoque hacia el desarrollo competitivo de la resolución de problemas.

El quinto factor denominado Debilidades, está relacionado con las acciones que el docente debe mejorar dentro del aula, según los estudiantes participantes. En este apartado, afirman que sus docentes orientan la clase bajo su propio criterio y no los tienen en cuenta a ellos para sugerencias y observaciones. En menor medida, atribuyen cierta dificultad para explicar efectivamente a todo el curso al mismo tiempo, a pesar de su esfuerzo. Este comportamiento de los docentes no es lo que se espera, según el MEN (2002), se requiere que el docente incorpore sus experiencias y saberes en estrategias didácticas bajo las condiciones pedagógicas (curriculares, didácticas y evaluativas) permitiendo a sus estudiantes propiciar escenarios dinámicos en torno a trabajos en equipo, propiciando espacios para lograr diálogos de intercambio conceptual entre estudiantes y el profesor con el objeto de lograr aprendizajes significativos, integrando el contexto escolar (institucional) y extraescolar (sociocultural) de los estudiantes.

El sexto factor denominado Recursos, hace referencia los medios físicos utilizados como herramienta didáctica en el desarrollo del tema. Estos recursos pueden ser tradicionales o tecnológicos para un mejor desempeño dentro del aula y la evaluación. Actualmente, son muy pocos los docentes del área de matemáticas que implementan nuevos recursos especialmente tecnológicos hacia el aprendizaje de las matemáticas.

\section{Conclusiones}

Se les debe reconocer a los docentes de matemáticas su gran compromiso y sentido de pertenencia en su quehacer pedagógico y el gran dominio conceptual hacia los contenidos curriculares del área de matemáticas en cada uno de los grados de enseñanza a nivel institucional; sin embargo los resultados reflejados en el instrumento de investigación son acordes con el pensamiento de Ruiz, L. L. W., y Pérez, M. I. R. (2014), debido a las falencias que actualmente se están presentando durante el desarrollo de la práctica pedagógica es la falta de coherencia y una permanente actualización hacia la didáctica y el uso de herramientas tecnológicas con la finalidad de despertar el interés de aprendizaje en los estudiantes y especialmente evitar un bajo rendimiento académico.

El quehacer del profesor de matemáticas en su proceso de enseñanza aprendizaje enmarca su preocupación específica hacia el cumplimiento del contenido curricular sin presentarse una coherencia hacia la formación del pensamiento competitivo del estudiante entorno a los estándares planteados por el MEN; las evaluaciones que aplican los docentes aún siguen siendo tradicionalistas, realizadas a partir del desarrollo de talleres, guías y textos que debe aprender el estudiante.

Estas actividades no son las esperadas por el MEN, puesto que el docente debe incorporar sus experiencias y saberes en estrategias didácticas bajo las condiciones pedagógicas 
Eco.Mat

Cúcuta-Colombia

Vol. 6

No. 1

Enero-Diciembre 2015

ISSN 1794-8231

ISSN 2462 - $8794 \mathrm{We}$

PP: 22-33
Aprendizaje y formación por competencias del pensamiento numérico

que permitan propiciar escenarios dinámicos en torno a trabajos en equipo logrando espacios para diálogos de intercambio conceptual entre estudiantes y profesores; esto permitirá que el estudiante se fortalezca hacia un pensamiento basado en las competencias, logrando un aprendizaje significativo integrando el contexto escolar (institucional) y extraescolar (sociocultural).

Es por esta razón, que el aprendizaje de la competencia numérica según los resultados de la investigación, muestran una enseñanza en forma mecánica y rutinaria con muy poca actividad hacia temas vinculados hacia la vida real, el desarrollo de proyectos en torno a los temas del área de matemáticas especialmente los números enteros y este tema a su vez ser vinculado en las otras áreas de formación del estudiante, la resolución de problemas y la relación del pensamiento numérico con el entorno; así como lo afirma, Giménez (2012) la realidad escolar es que los profesores del área de matemáticas desarrollan el aprendizaje de los números en forma algorítmica, desprovistos del mundo real y esto debilita la formación del pensamiento competitivo que requieren los estudiantes.

Para ser competente numéricamente el quehacer pedagógico de los docentes de matemáticas como lo manifiesta Giménez (2012), no se pueden reducir al simple uso de los números (alfabetismo numérico), como tampoco debería llevar únicamente el desarrollo de estructuras numéricas (algebrísmo descontextualizado). Entre ambos, se evade a veces el elemento clave de la competencia numérica que es el cuidado del contexto en el diseño de actividades matemáticas.

Además, los resultados del instrumento según los estudiantes, manifiestan que el profesor solo tiene en cuenta su criterio y experiencia para desarrollar las clases y en muy pocas oportunidades considera los resultados de las evaluaciones que permita implementar nuevas estrategias; como lo manifiesta el MEN (2002), las competencias deben estar relacionadas directamente con los saberes y conocimientos requeridos para la ejecución de actividades didácticas propias de una disciplina particular.

\section{Referentes}

Alliaud, A. (1997). ¿Quiénes eligen hoy ser maestros en Argentina? Perspectivas: Revista trimestral de educación comparada, (1), (pp.165-177).

Bruno A. (2007). La enseñanza de los números negativos: aportaciones de una investigación. Números. Revista de didáctica de las matemáticas. Consultado el 17 de junio de 2013. Recuperado de http://www.sinewton. org/numeros/numeros/29/Articulo01.pdf

Bruno, A. (1997). La enseñanza de los números negativos: formalismo y significado. Consultado el 16 de octubre del 2014. Recuperado de http://dmle.cindoc.csic.es/ pdf/GACETARSME_2001_04_2_05.pdf

Cid, E. (2000). Obstáculos epistemológicos en la enseñanza de los números negativos. Consultado el 16 de octubre del 2014. Recuperado de http://www.ugr.es/ jgodino/ siidm/cangas/Negativos.pdf

Delors, J. (2013). Los cuatro pilares de la educación. Galileo, (23). Consultado el 31 de marzo del 2015. Recuperado de http://www. uv.mx/dgdaie/files/2012/11/CPP-DC-DelorsLos-cuatro-pilares.pdf

Fernández S. (2011). Análisis Factorial. Universidad Autónoma. Consultado el 20 de noviembre del 2014. Recuperado de http://www.fuenterrebollo.com/Economicas/ ECONOMETRIA/MULTIVARIANTE/ FACTORIAL/analisis-factorial.pdf

Gallardo,A.(2002). Theextensionofthenaturalnumber domain to the integers in the transition from arithmetic to algebra. Educational Studies in Mathematics, 49(2), (pp.171192). Consultado el 31 de marzo del 2015. Recuperado de http://link.springer.com/ article/10.1023/A:1016210906658 
Giménez (2010). Potenciando competencia numérica con alumnado de 6 a 12 años. Consultado el 31 de marzo del 2015. Recuperado de https://activitat-matematica. wikispaces.com/file/view/gimenez+nombres. pdf

González, J. L., Iriarte, M., Jimeno, M., Ortíz, A., Ortíz, A., Sanz, E. \& Vargas Machuca, I. (1999). Números enteros. Matemáticas: cultura y aprendizaje. pp. 27- 157. Madrid: Editorial Síntesis, S.A.

Hernández Sampieri, R., Fernández Collado, C., \& Baptista Lucio, P. (2010). Metodología de la investigación. México: Editorial Mc Graw Hill.

Ministerio de Educación Nacional - MEN (2012). Evaluación de Competencias para el ascenso o reubicación de nivel salarial en el escalafón docente de los docentes y directivos docentes regidos por el decreto Ley 1278 de 2002. Consultado el 03 de marzo del 2015. Recuperado de http://www.mineducacion. gov.co/proyectos/1737/articles-310888_ archivo_pdf_filosofia.pdf

Ministerio de Educación Nacional - MEN (2006). Estándares Básicos de Competencia en Matemáticas. Consultado el 16 de octubre del 2014. Recuperado de http://www. mineducacion.gov.co/1621/articles-116042 archivo_pdf2.pdf

Myers, Robert (1999): Atención y desarrollo de la primera infancia en Latinoamérica y El Caribe: Una revisión de los diez últimos años y una mirada hacia el futuro, en Revista Iberoamericana de Educación, n. ${ }^{\circ}$ 22, (pp. 17-39), Madrid, OEI. Consultado el 31 de marzo del 2015. Recuperado de http://www. rieoei.org/rie22f.htm

Orton, A. (1990). Didáctica de las matemáticas: cuestiones, teoría y práctica en el aula (Vol. 14). Ediciones Morata. Consultado del 30 de marzo del 2015. Recuperado de: https://books.google.es/boo $\mathrm{ks}$ ?hl=es\&lr=\&id=DWBH5HdniK4C\&oi= fnd \&pg $=$ PA $9 \& d q=$ did $\%$ C $3 \%$ A 1 cticas + ma tematicas\&ots $=8 \mathrm{ZtmPxZnXR \& sig=Ypgw-}$ 2WI4rc8eSDq1SkHTtDjPC0

Osorio, G., \& Fernando, L. (2014). Los números enteros negativos en la matemática moderna y la matemática actual (Doctoral dissertation). Consultado el 31 de marzo del 2015. Recuperado de http://bibliotecadigital. univalle.edu.co/handle/10893/7705

Pardo M., A \& Ruiz D, M. A. (2002). SPSS V21 Guía para el análisis de datos. Editorial Madrid. Editorial McGraw Hill.

Peñuelas, M. A. R. (2010). Métodos de investigación: diseño de proyectos $y$ desarrollo de tesis en ciencias administrativas, organizacionales $y$ sociales. Universidad Autónoma de Sinaloa.

Rico, R. (2004). Evaluación de competencias matemáticas: proyecto PISA/OCDE 2003. In Investigación en educación matemática: Octavo Simposio de la Sociedad Española de Investigación en Educación Matemática (SEIEM): A Coruña, 9-11 septiembre 2004 (pp. 89-102). Servicio de Publicaciones. Consultado el 01 de abril del 2015. Recuperado de http://dialnet.unirioja.es/ descarga/articulo/1017761.pdf

Ruiz, L. L. W., \& Pérez, M. I. R. (2014). Factores que influyen en los alumnos para que no se encuentren motivados en la clase de matemáticas y qué papel juega el docente como agente motivador. In Congreso Virtual sobre Investigación Educativa. Consultado el 30 de marzo del 2015. Recuperado de http:// cenid.org.mx/cied_2014/memorias/index. php/CIED/article/download/25/25

Sánchez E., Hoyos V. \& López G. (2011). Sentido numérico y pensamiento algebraico. En Aprendizaje y enseñanza de las Matemáticas Escolares: Casos y Perspectivas. (pp. 37-58). Consultado el 31 de marzo del 2015. Recuperado de http://basica.sep.gob. mx/MATEMATICAS\%20web.pdf. 